\title{
Aprendizaje-servicio en contextos de confinamiento y pandemia: sostener la relación Universidad-Comunidad mediante la presencia virtual
}

\section{José Santiago Andrade-Zapata}

Pontificia Universidad Católica del Ecuador, Ecuador

\section{Ana Luisa López-Vélez}

Universidad del País Vasco, España

\section{Resumen}

La presente experiencia cuenta el modo en que el proyecto "Luli en Servicio 2020" vivió el trance de la pandemia y el confinamiento dejándose afectar por la realidad, respondiendo a ella desde las capacidades digitales en favor de una campaña de seguridad alimentaria para personas en situación de vulnerabilidad de muy bajos ingresos, desde la toma de decisiones, la organización propia en manos de los estudiantes y la capacidad de alianzas, hasta la rendición de cuentas. Con las lecciones aprendidas se retomó la idea que animó al principio al grupo: trabajar con una comunidad remota de la costa ecuatoriana.

\section{Palabras clave}

Aprendizaje-servicio, toma de decisiones, competencias. Iberoamericana de Aprendizaje Servicio, 10, 89-100. DOI10.1344/RIDAS2020.10.8 


\title{
Service-learning within the context of a pandemic and a lockdown: sustaining University-Community relationship through virtual presence
}

\begin{abstract}
This paper reports how the 'Luli in Service 2020' service-community project experienced the critical moments of the pandemic and lockdown. The project did not shy away from reality, but rather responded to it by using existing digital capabilities in a campaign aimed at providing food security for low-income, vulnerable people, and in all stages of the campaign: from decision-making, empowering students to selforganize and forming alliances with partners to providing accountability. With the lessons learned, the idea that motivated the group at the beginning was retaken: working with a remote community on the Ecuadorian coast.
\end{abstract}

\section{Keywords}

Service-learning, decision making, competences. 


\section{Introducción}

El aprendizaje-servicio hace realidad su sentido en la presencialidad y en la experiencia misma de situarse en una localidad: un lugar distinto al de la Universidad en el que la formación, la investigación y una docencia renovada encuentran pertinencia, correspondencia y razón al cooperar por la calidad de vida para las personas de dicho lugar (Aramburuzabala, Cerrillo y Tello, 2015).

Diferentes y muy variados tipos de implementación representan las formas en que el aprendizaje-servicio se desarrolla en el contexto universitario. En el caso del aprendizaje-servicio en el proyecto Luli en Servicio del Programa de Liderazgo Ignaciano Universitario Latinoamericano (PLIUL-LULI) de la Pontificia Universidad Católica del Ecuador (PUCE), puede determinarse como una experiencia cocurricular, pues se implementa una vez desarrollada la parte curricular $y$, a periodo académico siguiente, se implementa el proyecto (Opazo, 2015). El curso y su experiencia posterior de servicio está abierto a todas las carreras de la universidad; $y$, tanto la materia como el proyecto en sí, acreditan valor curricular en créditos y en horas de servicio comunitario, respectivamente; estas últimas superan en 3 y 4 veces el requisito de la normativa. Esta iniciativa había desarrollado ya una relación con la comunidad del litoral ecuatoriano de San Isidro, en la vasta provincia de Manabí a $300 \mathrm{~km}$ de la ciudad de Quito, sede de la universidad.
El equipo de estudiantes está conformado por 13 estudiantes repartidos de la siguiente manera:

Tabla 1. Equipo Luli en Servicio composición por género y número

\begin{tabular}{|ccc|}
\hline Género & Femenino & Masculino \\
Número & 8 & 5 \\
\hline
\end{tabular}

Fuente: elaboración propia

Tabla 2. Equipo Luli en Servicio: composición por carrera y año/estado de avance.

\begin{tabular}{|c|c|c|}
\hline Carrera & $\mathbf{N}^{\circ}$ & $\begin{array}{c}\text { Semestre de } \\
\text { carrera/estado }\end{array}$ \\
\hline $\begin{array}{c}\text { Psicología Clínica } \\
\text { (10 semestres) }\end{array}$ & 7 & 1 - egresada \\
\cline { 3 - 3 } & & $2-9^{\circ}$ nivel \\
\hline $\begin{array}{c}\text { Sociología } \\
\text { (8 semestres) }\end{array}$ & 2 & $8^{\circ}$ semestre \\
\hline $\begin{array}{c}\text { Gestión Social } \\
\text { (8 semestres) }\end{array}$ & 1 & $8^{\circ}$ semestre \\
\hline $\begin{array}{c}\text { Jurisprudencia } \\
\text { Jurisel }\end{array}$ & 1 & Graduado (2019) \\
\hline $\begin{array}{c}\text { Administración de } \\
\text { Empresas }\end{array}$ & 1 & Graduada (2020) \\
\hline $\begin{array}{c}\text { Lenguas Aplicadas } \\
\text { en Negocios y } \\
\text { Relaciones } \\
\text { Internacionales }\end{array}$ & 1 & 1 - egresada \\
\hline
\end{tabular}

Fuente: elaboración propia

A la fecha se habían realizado ya, entre 2018 y 2019 , dos intervenciones de campo con cohortes diferentes. Con esa experiencia, se concebía una tercera para este 2020 con relación a la recuperación de la cultura montubia (de los pobladores del campo costero). 
Por una parte, en el verano de 2018, por espacio de dos semanas, una serie de talleres vespertinos de refuerzo escolar en las comunidades de Pechichal y Río Mariano Tutumbe. Espacios de recreación donde la propuesta lúdica implicaba el manejo de la frustración del impulso violento al desarrollo de la capacidad de expresión verbal. Por otra parte, en el verano de 2019, se replicó el mismo esquema de intervención con ligeros ajustes y modificaciones. En este 2020, el nuevo equipo se planteó el apoyo a la causa cultural, iniciando este 2020, con dos visitas entre enero y febrero 2020, consistentes en un taller de tres días en el contexto del verano costeño (febrero-abril), con una intervención puntual de recreación y la concepción de un plan mensual de trabajo para levantar entrevistas a los adultos mayores en el marco de la recuperación de la cultura montubia que lleva adelante la fundación Raíces y Sueños. Por tanto, se preveía un año intenso y fructífero, hasta que llegó el confinamiento. La pandemia quebró dicha modalidad y hubo un trance necesario.

El presente artículo reflexiona sobre el tránsito de un proyecto estrictamente presencial, de campo, a una fórmula donde la virtualidad encuentra su lugar, a propósito de la colaboración en una experiencia concreta para la seguridad alimentaria a personas y colectivos de economía de calle, liderada por la ONG CMT, en Quito y las lecciones aprendidas para retomar la relación con San Isidro a partir de esta experiencia. Por tanto, la pregunta de arranque es: ¿de qué manera el proyecto Luli en
Servicio, mediante la tecnología, respondió a los retos de la pandemia para realizar su trabajo con la comunidad?

Con ello buscamos principalmente establecer los parámetros que determinaron el paso de una modalidad presencial a una modalidad virtual en el desarrollo de las actividades del proyecto Luli en Servicio del Programa de Liderazgo Ignaciano Universitario Latinoamericano de la PUCE, Ecuador.

Para lograr esta meta será necesario identificar los desafíos propios de la iniciativa y sus énfasis a propósito de la pandemia; determinar el proceso de toma de decisiones desde el impacto del confinamiento a la iniciativa de seguridad alimentaria y la retoma de la relación con San Isidro y el proyecto original; y finalmente, establecer las posibilidades que desarrollaron las y los estudiantes en relación a la virtualidad.

\section{El impacto de la pandemia y el confinamiento}

Como todas las instituciones, la Pontificia Universidad Católica del Ecuador (PUCE) pasó, de un momento a otro, al confinamiento, como consecuencia de la pandemia. Sus puertas se cerraron y sus actividades pasaron a modo remoto a partir del 13 de marzo de 2020.

Este salto a la modalidad remota supuso un elemento disruptivo en la lógica presencial, conforme lo señala Arriagada (2020) "las instituciones de educación han tenido que ampliar sus redes tecnológicas para cubrir las innumerables actividades y demandas 
que se deben seguir desarrollando" (p.2). El uso de videoconferencias, incorporación de mayor número de recursos por medio de los entornos virtuales de aprendizaje, representó un salto con importantes lecciones que han supuesto, a esta parte del camino, implementación de mayores y mejores criterios y opciones en su uso e incidencia en el marco formativo de una Institución de Educación Superior (IES).

En el ámbito de la vinculación con la colectividad de la PUCE, el servicio se centró en la atención y apoyo en el área de la salud: pruebas PCR, atención con estudiantes de especialidades médicas, de nivel de posgrado, producción de escudos faciales con impresoras 3D y la activación de diversas iniciativas remotas permitieron replantear la tarea de vinculación en varios sentidos. En estos tiempos de desafíos y emergencia se legitima este tipo de conocimiento basado en el servicio, cuya finalidad es contribuir con soluciones a los problemas de la sociedad y el medio ambiente, y de esta manera, se contribuye a la transformación social (Aramburuzabala, 2013). La pandemia ha agravado las desigualdades en todo orden (ITESO - Universidad Jesuita de Guadalajara, 2020).

Un elemento determinante para sostener la relación UniversidadComunidad fue la comunicación remota. El equipo Luli en Servicio y la comunidad de San Isidro recurrieron desde las llamadas telefónicas, el uso de WhatsApp hasta el descubrimiento de Zoom, el uso de redes sociales marcó la diferencia entre un momento de silencio por el impacto inicial del confinamiento hasta el inicio de nuevos modos y mecanismos de trabajo.

- "29/5/20. 5:30 - INV: Gracias, SC1. ¿Cómo estás? ¿Qué tal van las cosas por allí?

- 29/5/20. 5:33 - SC1 San Isidro: Hola INV Buenos días gusto saludarte Pues aquí todo está muy paralizado muy estancado sin perspectivas de futuro con suerte en San Isidro Pues hay algún contagio pero no se ha extendido demasiado pero seguimos en el aislamiento y por ahí como van.

- 29/5/20. 5:37 - INV: ¿Hay ya contagio? Pensé que lo remoto iba a significar una barrera [...] Por lo demás, pensándoles. Los muchachos que hicieron su intervención en el verano anterior, todavía ilusionados: quieren algún contacto con sus conocidos de san Isidro para saber cómo están. Eso.

- 29/5/20. 5:38 - INV: https://youtu.be/XXXXXXXX

- 29/5/20. 9:35 - SC1 San Isidro: Gracias INV por toda la información Disculpa que andaba ocupado en San Isidro hubo como tres fallecidos personas mayores que habían llegado de fuera y ahora y como 8 días que eran familiares de ellos y alguno más que están controlados en cuarentena de momento no se ha propagado más estamos aislados con ganas de continuar con muchos planes en nuestro proyecto y ya entiendo todo lo 
que me dices de las dificultades en la ciudad la universidad ya leí el recorte de los presupuestos eso es terrible ya estaba viendo también este vídeo En fin estamos en una crisis grande $y$ tendremos que ir saliendo poco a poco seguimos en comunicación un abrazo saludos a todos".

(Mensaje de WhatsApp del investigador a un socio comunitario. 20-04-2020. 20:49. Estudiante del equipo Luli en Servicio).

Para el caso del proyecto Luli en Servicio, la brecha comunicativa con su iniciativa en la costa dio lugar, en ese instante, a una experiencia de apoyo en la promoción a una iniciativa que provee alimentación a personas en situación y economía de calle en Quito, consistente en la entrega de alimentos de primera necesidad con frecuencia semanal. Hubo tres momentos sustanciales en el proceso: enfrentar el impacto de la pandemia y el confinamiento como grupo; establecer una estrategia en el corto plazo; y emprender nuevamente con el proyecto en ciernes, sobre la base de las lecciones aprendidas.

- "Hola chicos, en la reunión llegamos a ciertos puntos claves:

1) Decidimos apoyar al CMT en una campaña publicitaria para recaudación de fondos, esto se gestionará a través de canales formales dentro de la PUCE.

Y... básicamente eso :3

Linda noche :D"
(Mensaje de WhatsApp de un estudiante del proyecto Luli en Servicio. 20-04-2020. 20:49. Estudiante del equipo Luli en Servicio)

A consecuencia del COVID-19 se redujo la comunicación solamente a mensajes de WhatsApp con actores de San Isidro. Se procuró una comunicación básica situada en preguntar por la situación en la zona y el estado de ánimo de las personas socias y sus familiares; igualmente se mantuvo la comunicación con autoridades de la zona.

Otro elemento sustancial era la incertidumbre. Ni el tiempo, ni las posibilidades de desplazamiento eran favorables, puesto que las noticias y el escenario no daban datos del final frente al paso del tiempo y el retorno a la normalidad (ahora sabemos que nunca será como antes).

Un tercer elemento fue la argumentación al interior del grupo. El primer argumento fue la necesidad de actuar de alguna manera frente a la situación crítica de personas vulnerables o en situación de desigualdad. El segundo argumento fue la oportunidad de actuar en la promoción de la campaña para sostener la entrega de kits alimentarios en barrios urbanos marginales donde habitan personas en situación de calle en Quito. Esto último fue acogido por el grupo como una suerte de pausa frente a la posibilidad primera de continuar oportunamente el contacto con San Isidro.

Un cuarto hecho fue, precisamente, la campaña de promoción a donaciones 
para la seguridad alimentaria. La gestión determinó, pues, una suerte de proyecto puntual de intervención donde los estudiantes implicados en el proyecto Luli pusieron a prueba sus capacidades organizativas, de trabajo en equipo, de gestión y de manejo de las redes sociales.

Esta propuesta tuvo correspondencia con la función sustantiva de la universidad quería responder a la demanda que representaba ( $y$ aun hoy representa) de comprender una realidad y hacer frente a ella, en el entendido de que una de las funciones sustantivas de la universidad es precisamente la vinculación y el modo en que ésta responde de manera integral y convergente en las demás funciones a la respuesta de la sociedad a los problemas que tiene que resolver, en el marco de justicia social que la PUCE tiene como marco de respuesta a estos contextos y sus demandas (Yépez-Reyes, González y García, 2020).

Esta propuesta quiso responder a la demanda que representaba y representa que el alumnado comprenda una realidad y le haga frente. Se respondió así a una de las funciones sustantivas de la universidad que es la vinculación y el modo en que ésta responde de manera integral y convergente en las demás funciones de la PUCE en el marco de la justicia social y la respuesta a los problemas de la sociedad (González, Yépez-Reyes y García, 2019).

\section{La toma de decisiones y actuación en el nuevo contexto}

El aprendizaje-servicio encuentra cauce en la experiencia que ofrece oportunidades de aprendizaje para sus actores. Desde la universidad se centra en el proceso de aprendizaje de las y los estudiantes; desde la comunidad en la experiencia colaborativa que ella representa. EI COVID-19 supuso una experiencia no planificada desde todo orden. Arremetió con el componente de incertidumbre en un marco en el que, desde la seguridad del currículo, el aprendizaje-servicio demanda una planificación y unas condiciones de posibilidad expresadas en el lenguaje de las y los estudiantes que establecen el carácter de esta vivencia no prevista.

En la línea de Dewey (1938) y la forma en que adopta la comprensión de la Educación Progresiva, situada en una realidad de oposición a la Educación Tradicional, situada en roles fijos y organizados en la institución social que es la escuela, la Progresiva encuentra en el contacto con situaciones reales su razón y naturaleza y va más allá de los patrones establecidos de reglas, tiempo y espacio (Dewey, 1938).

Efectivamente, este contexto de pandemia y confinamiento nos hacen repensar nuestras habituales formas de realizar el aprendizaje-servicio, con un margen muy angosto para responder. Ciertamente de la década del 30 del siglo pasado a la fecha, ha corrido mucha agua, pero, indiscutiblemente nos permite ver un escenario que fue sustancial en la obra de Dewey: una crisis a la que las mismas personas debían responder y donde la academia, 
más allá de la salud y la economía se miró de manera distinta: entre conectarse con la realidad o mantenerse en su institucionalidad.

Por otra parte, nos permite volver a las fuentes de lo que significa el aprendizaje-servicio. Esto tiene que ver con una de las características del proyecto, y del mismo programa de liderazgo ignaciano. Es decir, ante la incertidumbre y la falta de opciones, ante el trauma del fenómeno de la pandemia, hubo la necesidad de volver al punto de partida. En lenguaje ignaciano, volver al Principio y Fundamento (Loyola, 1952), cuya pregunta sustancial fue ¿qué razón de fondo da sentido a un liderazgo como el aprendido? Elemento que fue verbalizado en el grupo en un contexto de encuentro y resignificación de lo vivido: el servicio para el cuidado de la dignidad humana y la respuesta a las desigualdades, como señala una de las estudiantes del proyecto "hay factores subyacentes que generan desigualdades" y a continuación expresa su opción frente al proyecto: "parte del a experiencia de Luli es dar cuenta de esas desigualdades"

(Andrade-Zapata, 2020).

\section{EI proceso de actuación y respuesta}

El proceso duró de abril a mayo de 2020, con las siguientes fases:

\section{a) La toma de decisión}

Frente al escenario de la pandemia, hubo dos momentos en los que los estudiantes debatieron sobre su propia posibilidad de activarse en relación a la idea primera de trabajo en la comunidad de la costa ecuatoriana, en San Isidro.

El segundo momento fue hablar sobre la motivación y el sentido del grupo con una identidad propia y un carácter orientado al servicio desde un liderazgo comprometido y atento. En ese espacio, cuya metodología promovió la palabra de los participantes, se definió la alternativa de ayudar en la promoción a la campaña \#QuédateEnCasa como estrategia para sostener a la población en situación de calle en Quito frente a la expansión de la pandemia que para efectos de conteo en el tiempo que duró la misma se repartieron 50 toneladas de alimentos (AndradeZapata, 2020).

De los doce estudiantes, tres expresaron su desacuerdo, pero se sumaron a la iniciativa. Algunos de los argumentos contemplaban el cambio de iniciativa, el diferir de la realización del proyecto hasta volver a la normalidad, entre otras.

Finalmente, se optó por un trabajo íntegro de promoción que duraría los meses de abril a mayo de 2020 mediante la activación en redes sociales, producción de contenidos y productos comunicacionales, apalancamiento en la fanpage del programa de Liderazgo.

b) Diseño del plan de promoción de la campaña

El plan contempló la publicación de productos publicitarios de sensibilización sobre la base de un marco ligado a datos duros producidos 
por agencias internacionales, autoridades del mundo productivo y del trabajo.

Cada post dirigido especialmente a público juvenil pero también a adultos profesionales, por ser público característico de la comunidad universitaria, debería llevar un mensaje objetivo en el marco del efecto de la pobreza, justicia y desigualdades en población en situación de vulnerabilidad y riesgo. Por otra parte, guardaría la línea gráfica del programa de Liderazgo Ignaciano y se dirigiría a la audiencia que este tiene ya en redes sociales. Se designaron responsables de contenidos y creatividad, así como responsables de la elaboración de las piezas publicitarias para las redes.

El sentido de la campaña invitó a una sensibilización desde la justicia y con claro respeto a la dignidad de las personas. Las ilustraciones 1 y 2 del Programa de Liderazgo Ignaciano Universitario Latinoamericano llevan adelante este carácter.

Ilustración 1. Post promocional con información socioeconómica

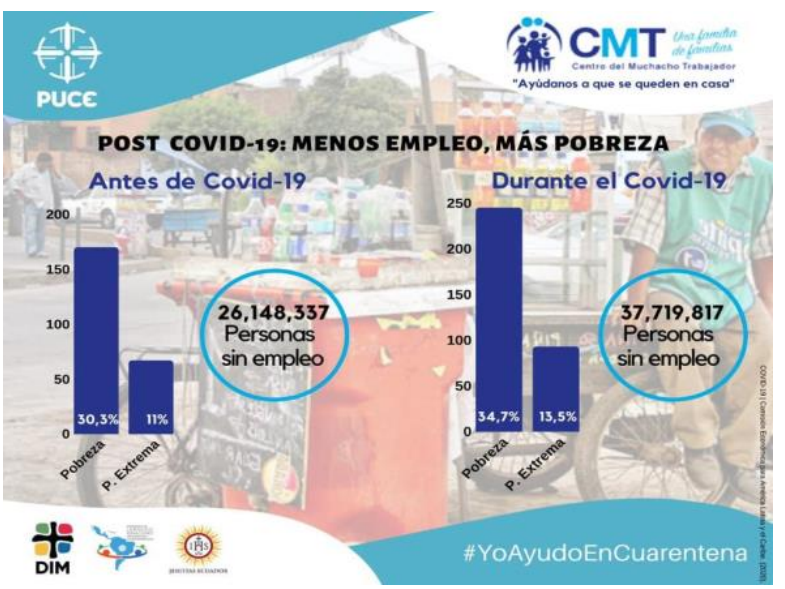

Fuente: elaboración propia

Ilustración 2. Post promocional con información socioeconómica

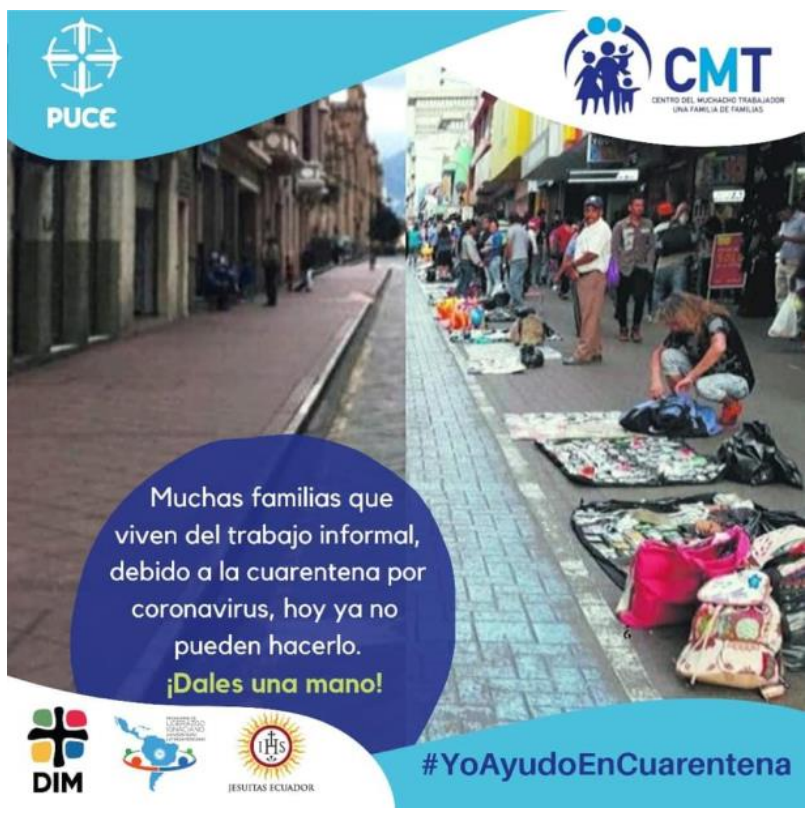

Fuente: elaboración propia

\section{c) Estrategia de adhesiones para la campaña}

El equipo Luli en Servicio activó una serie de comunicaciones que movilizaron a que varias asociaciones de estudiantes de la universidad realizaran donativos en dinero, y en otros casos, llevó a activar otro tipo de iniciativas, por ejemplo la PUCE Solidaria en la que confluyen esfuerzos de atención a población migrante en situación de calle en el orden de la salud, emprendimiento, seguridad alimentaria; colaboración con proyectos de vinculación en la zona rural como es Chugchilán (González, Yépez-Reyes y García, 2019). 
La ilustración 3 recoge estas colaboraciones.

Ilustración 3. Post de agradecimiento a las asociaciones participantes

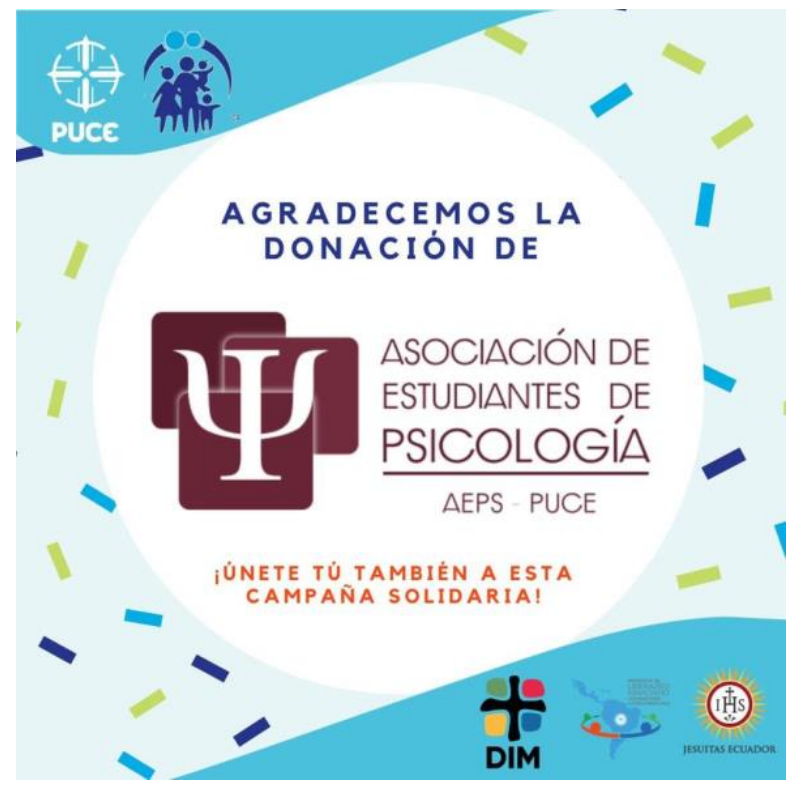

Fuente: elaboración propia

Esto significó un registro de alianzas promovidas desde y entre estudiantes en la promoción de esta iniciativa.

Fueron 11 asociaciones de estudiantes las que participaron con donativos en metálico, al mismo tiempo de replicar y agradecer públicamente su respuesta.

\section{d) Evaluación y cierre de la campaña}

Al concluir el plazo establecido, que se extendió una semana más por efectos del mismo proceso, se realizó una sesión de rendición de cuentas y recuento. El testimonio fue también recogido en redes sociales, con las lecciones y aprendizajes vividos y adquiridos, por ejemplo, la capacidad organizativa, la convicción ética que movió al equipo, la capacidad de acuerdos con diversos actores y la misma capacidad de aplicar su saber digital y según otra estudiante "de mis (sus) círculos sociales para una causa", para un fin social (Andrade-Zapata, 2020).

El proceso constituyó, para el alumnado universitario, en un espacio de tránsito desde una actividad concreta de servicio y derivó en un aprendizaje que permitió que los y las estudiantes universitarias participantes en el proyecto Luli dieran una respuesta concreta a una urgencia del contexto, y tuvieran la oportunidad de poner a prueba las capacidades de organización y comunicación propias de una experiencia de aprendizaje-servicio.

\section{Conclusiones}

¿De qué manera el proyecto Luli en Servicio, mediante la tecnología, respondió a los retos de la pandemia para realizar su trabajo con la comunidad?

La respuesta sería mediante el acceso inteligente a la tecnología y las Redes Sociales. Es decir, hubo elementos determinantes: una decisión de equipo, la capacidad de respuesta a la incertidumbre, la recursividad propia de las y los estudiantes en ese ámbito que les es tan natural en las Redes Sociales y la determinación de retomar con este tránsito, su vínculo primero con la comunidad de San Isidro.

Participación de los estudiantes en la toma de decisiones. El rol protagonista de los estudiantes en proponer y 
asumir proyectos. Es decir, el aprendizaje-servicio tiene su centralidad en ellos, en el marco de una relación Universidad-Comunidad. Dicho rol es activo y se orienta de manera esencial en la toma de decisiones. ¿Qué tanto los estudiantes se anclan a iniciativas adultocéntricas o van tejiendo como parte de un equipo la definición y ejecución del proyecto en sí mismo? Para el caso, está claro que las condiciones de actuación del alumnado implicado estimulan a que se promueva la toma de decisiones y la implicación desde la propia opción, un marco motivacional, un proceso formativo realizado y certificado y un marco de referencia frente a las posibilidades y coyunturas permiten y mueven a los estudiantes a enfrentar la tarea de considerar y decidir qué y cómo responder a una demanda. $Y$ esto en el marco institucional, con los ritmos institucionales que no siempre son ágiles como los personales, pero aseguran el respaldo correspondiente.

Capacidad de respuesta ante la incertidumbre. La coyuntura supone una demanda y frente a ella, el grupo asume una posición de verbalizar lo que esta situación -al efecto por el COVID19- representa y pide. Hubo, por tanto, un ejercicio de toma de decisiones desde la persona, el grupo, los socios y la coyuntura. El efecto de la pandemia no solo representa un impacto en una idea preliminar. Para el caso, el proceso fue, esperar $y$, hasta tanto, actuar en el radio de posibilidades que se tenían. Eso motivó a la consideración y posterior decisión de apoyar a la campaña de seguridad alimentaria.
La tecnología en el aprendizaje-servicio. El promedio de edad de los participantes se sitúa entre los 21 y 26 años. Esto supone su condición de nativos digitales $y$, por tanto, el uso de tecnologías de información y comunicación (TIC) les es absolutamente propio. Sin embargo, el criterio y alcance de uso redunda en nuevos horizontes. Son competencias aplicadas en las TIC para la resolución de problemas reales y en donde el tejido social se asienta, en buena medida en el uso de redes sociales. Las tecnologías hoy conectan sincrónicamente a los actores y es posible una presencialidad virtual; por tanto, la garantía de conectividad representa una condición sustancial a la hora de establecer un proceso colaborativo.

Con esto en la mochila, retornamos a san Isidro en cuya campaña "Internet para Pechichal" pondremos a prueba lo aprendido en esta experiencia.

\section{Referencias bibliográficas}

Andrade-Zapata, J. (2020). Luli en Servicio - Recuento [Archivo de video]. Recuperado de:

https://drive.google.com/file/d/1uSVW BrY0B921YQTALJEZ7kzXo0SBZe7A/view ?usp=sharing

Aramburuzabala, P. (2013). Aprendizaje-servicio: una herramienta para educar desde y para la justicia social. Revista Internacional de Educación para la Justicia Social, 2(2), 5-11. Recuperado de: https://revistas.uam.es/riejs/article/vie $\mathrm{w} / 369 / 359$ 
Aramburuzabala, P., Cerrillo, R., y Tello, I. (2015). Aprendizaje-servicio: una propuesta metodológica para la introducción de la sostenibilidad curricular en la Universidad.

Profesorado. Revista de Currículum y Formación de Profesorado, 19(1), 7895. Recuperado de:

https://www.ugr.es/ recfpro/rev191AR $\underline{\text { T5.pdf }}$

Arriagada, F. (2020). El desafío de la vinculación con el medio en tiempos de pandemia COVID-19. Ciencia y

Enfermería, 26(11), 1-3.

https://doi.org/10.29393/ce26-

$\underline{4 d v f a 10004}$

Dewey, J. (1938). Experience and

Education. Nueva York, Estados Unidos de América: Touchstone.

González, J. C., Yépez-Reyes, V., y García, E. (Eds.). (2019). Vinculación con la Colectividad: una propuesta de gestión. Recuperado de:

https://edipuce.edu.ec/wpcontent/uploads/2019/08/Vinculacion C on la colectividad.pdf

ITESO - Universidad Jesuita de Guadalajara. (28 de septiembre de 2020). Pandemia y derechos humanos Aportaciones desde la ONU y las universidades jesuitas de América Latina [Archivo de Vídeo]. YouTube. https://www.youtube.com/watch?v=8 8tLLzj1Mg\&feature=youtu.be

Loyola, S. I. (1952). Ejercicios

Espirituales. Madrid, España:

Apostolado de la Prensa.

Opazo, H. (2015). Experiencias de aprendizaje-servicio en la formación del profesorado. Un estudio de caso (tesis doctoral). Universidad Autónoma de Madrid, Madrid, España. Recuperado de:

https://repositorio.uam.es/handle/1048 $\underline{6 / 670908}$

Yépez-Reyes, V., González, J.C., y García, E. (2020). Intérpretes de las necesidades de la comunidad. RIDAS, Revista Iberoamericana de Aprendizaje Servicio, (9), 142-154. doi:10.1344/RIDAS2020.9.9

Andrade-Zapata, J. y López-Vélez, A. (2020). Aprendizaje-servicio en contextos de confinamiento y pandemia: sostener la relación Universidad-Comunidad mediante la presencia virtual. RIDAS, Revista Iberoamericana de Aprendizaje Servicio, 10, 89-100. DOI10.1344/RIDAS2020.10.8 